Epidemiology and Infection

http://journals.cambridge.org/HYG

EPIDEMIOLOGY \&

INFECTION

Additional services for Epidemiology and Infection:

Email alerts: Click here

Subscriptions: Click here

Commercial reprints: Click here

Terms of use : $\underline{\text { Click here }}$

\title{
Nationwide surveillance study of human astrovirus infections in an Italian paediatric population
}

S. DE GRAZIA, V. MARTELLA, M. CHIRONNA, F. BONURA, F. TUMMOLO, A. CALDERARO, P. MOSCHIDOU, G. M. GIAMMANCO and M. C. MEDICI

Epidemiology and Infection / FirstView Article / January 2006, pp 1 - 5

DOI: 10.1017/S0950268812000945, Published online: 17 May 2012

Link to this article: http://journals.cambridge.org/abstract S0950268812000945

How to cite this article:

S. DE GRAZIA, V. MARTELLA, M. CHIRONNA, F. BONURA, F. TUMMOLO, A. CALDERARO, P. MOSCHIDOU, G. M. GIAMMANCO and M. C. MEDICI Nationwide surveillance study of human astrovirus infections in an Italian paediatric population. Epidemiology and Infection, Available on CJO 2012 doi:10.1017/S0950268812000945

Request Permissions : $\underline{\text { Click here }}$ 


\title{
SHORT REPORT
}

Nationwide surveillance study of human astrovirus infections in an Italian paediatric population

\author{
S. DE GRAZIA ${ }^{1 *}$, V. MARTELLA ${ }^{2}$, M. CHIRONNA ${ }^{3}$, F. BONURA ${ }^{1}$, \\ F. TUMMOLO ${ }^{4}$, A. CALDERARO ${ }^{4}$, P. MOSCHIDOU ${ }^{2}$, G. M. GIAMMANCO ${ }^{1}$ \\ AND M. C. MEDICI ${ }^{4}$ \\ ${ }^{1}$ Dipartimento di Scienze per la Promozione della Salute 'G. D’Alessandro', Università di Palermo, Palermo, Italy \\ ${ }^{2}$ Dipartimento di Sanità Pubblica e Zootecnia, Università Aldo Moro di Bari, Bari, Italy \\ ${ }^{3}$ Dipartimento di Scienze Biomediche, Sezione di Igiene, Università Aldo Moro di Bari, Bari, Italy \\ ${ }^{4}$ Sezione di Microbiologia, Dipartimento di Patologia e Medicina di Laboratorio, Università degli studi di Parma, \\ Parma, Italy
}

Received 26 January 2012; Final revision 11 April 2012; Accepted 14 April 2012

\section{SUMMARY}

The study investigated the genetic diversity of human astroviruses (HAstVs) detected in children hospitalized with gastroenteritis in Italy in 2008-2009. A total of 1321 faecal samples were collected in Parma (northern Italy), Bari (southern Italy), and Palermo (Sicily) and screened for the presence of HAstVs. RT-PCR amplification of a portion at the 5'-end of ORF2 allowed the detection of HAstVs in $3.95 \%$ of the patients. Four different genotypes (HAstV-1, HAstV-2, HAstV-4, HAstV-5) were found to be circulating during the study period, with HAstV-1 being the predominant type. Interestingly, a novel lineage, proposed as HAstV-2d, was found to have emerged in Parma in 2009. Investigating the genetic variability of HAstVs will be important for understanding the epidemiological trends and evolution of these viruses.

Key words: Astroviruses, epidemiology, gastroenteritis, virology (human).

Astroviruses (AstVs), family Astroviridae, are enteric viruses associated with gastroenteritis in young children in both developed and developing countries [1]. AstVs have a single-stranded positive-sense RNA genome containing three open reading frames (ORFs). ORF1a and ORF1b, at the 5 '-end of the genome, encode the non-structural viral proteins (serine protease and RNA-dependent polymerase), while ORF2, at the 3 '-end, encodes the capsid precursor protein. Human AstVs (HAstVs) are genetically and antigenically heterogeneous and include a

\footnotetext{
* Author for correspondence: Dr S. De Grazia, Dipartimento di Scienze per la Promozione della Salute 'G. D'Alessandro', Università di Palermo, via del Vespro 133, 90127 Palermo, Italy. (Email: simona.degrazia@unipa.it)
}

major group, classified into eight antigenic types (HAstV-1 to HAstV-8) [2] and a number of unusual AstVs (e.g. MLB1, HMO, VA) discovered recently and genetically more related to animal AstVs than to 'classical' HAstVs [3]. Sequence analysis of short fragments at either the 5'- or 3'-end of ORF2 and RT-PCR genotyping protocols with type-specific primers, have been used for genetic characterization of HAstV types 1-8 [4] and found to correlate with antigenic characterization with type-specific polyclonal or monoclonal antibodies [5]. Genotyping surveys have shown that HAst $\mathrm{V}-1$ is the most common type identified in children, followed by HAstV-2, -3, -4 and -5 [6]. Interestingly, upon molecular analysis of the ORF2, discrete sequence variation has been 
S. De Grazia and others

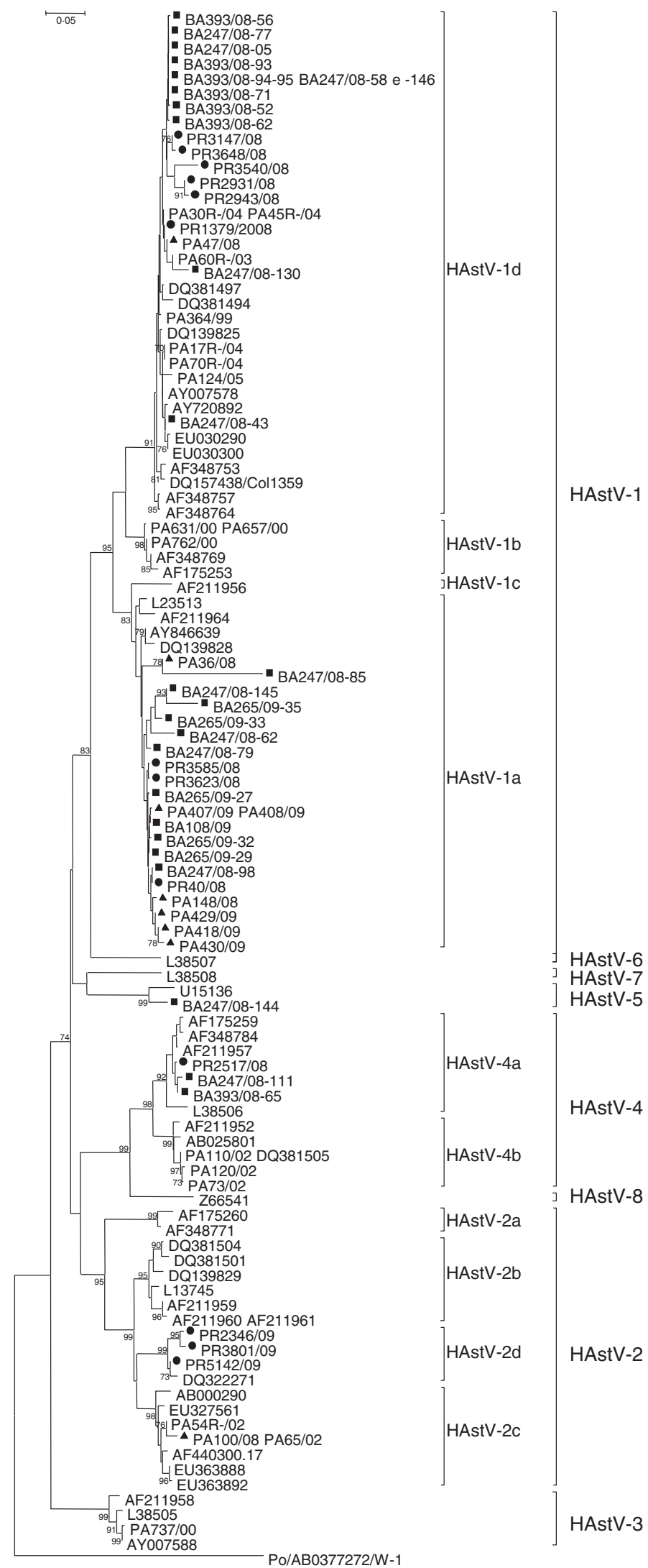

Fig. 1. Phylogenetical analysis of partial nucleotide sequences ( $348 \mathrm{bp}$ ) of HAstV ORF2 capsid region of 49 strains collected in Bari, Palermo, and Parma from 2008 to 2009. The Kimura two-parameter model of substitution and the neighbour-joining 
Table 1. Temporal and geographical distribution of HAstV genotype lineages

\begin{tabular}{llll}
\hline \hline Source & Year & Prevalence & HAstV genotype lineage detected $(n)$ \\
\hline Parma (northern Italy) & 2008 & $3 \cdot 87 \%$ & HAstV-1a (3), HAstV-1d (6), HAstV-4a (1) \\
& 2009 & $1 \cdot 38 \%$ & HAstV-2d (3) \\
Bari (southern Italy) & 2008 & $7 \cdot 42 \%$ & HAstV-1a (5), HAstV-1d (13), HAstV-4a (2), HAstV-5 (1) \\
Palermo (Sicily) & 2009 & $5 \cdot 8 \%$ & HAstV-1a (6) \\
& 2008 & $2 \cdot 6 \%$ & HAstV-1a (2), HAstV-1d (1), HAstV-2c (1) \\
\hline \hline
\end{tabular}

observed within each HAstV type. HAstV-1 has been classified into four lineages (1a-1d), while HAstV-2 and HAstV-4 have been classified into three $(2 \mathrm{a}-2 \mathrm{c})$ and two lineages $(4 \mathrm{a}, 4 \mathrm{~b})$, respectively [6, 7]. Predominant types and/or lineages appear to vary seasonally and geographically but only a few structured, nationwide epidemiological studies can be found in the literature. In addition, the use of different diagnostic and typing tools has generated heterogeneous data that are of difficult interpretation/ comparison. Surveillance studies conducted in Palermo, Italy, in 1999-2000 and 2002-2005, revealed that yearly prevalence of HAstVs in children hospitalized for gastroenteritis ranged from $0.5 \%$ to $7 \%$, similarly to the prevalence observed in other countries [7-9]. The predominant type circulating in the local population was HAstV-1 with temporal fluctuations of lineages $1 \mathrm{~b}$ and $1 \mathrm{~d}$. In 2002, the onset of new HAstV strains of types $2 \mathrm{c}$ and $4 \mathrm{~b}$ was observed.

In the present study, we extended the investigation on the prevalence and genetic diversity of HAstVs in Italy by enrolling laboratories located in three different geographical areas. From January 2008 to December 2009 a total of 1321 faecal samples, 693 in 2008 and 628 in 2009, were obtained from children aged $<5$ years, hospitalized with acute gastroenteritis. Of the 2008 samples, 258 were collected in Parma (northern Italy), 283 in Bari (southern Italy), and 152 in Palermo (Sicily), while in 2009 the samples from the three areas were 216, 103 and 309, respectively. The samples were screened for the presence of HAstVs by RT-PCR with HAstV-specific primers Mon269 and Mon270, amplifying a 348 nucleotide (nt) portion at the $5^{\prime}$-end of ORF2 [4]. The amplicons were sequenced and phylogenetical analysis was performed with CLUSTALW and MEGA 5.0 (www.megasoftware.net) software using a selection of reference sequences. The sequences of Italian HAstV strains have been deposited in GenBank under accession numbers: JQ434370-JQ434393 and JQ434395-JQ434397 (for Bari); JQ434362-JQ434369 and JQ434394 (for Palermo); JQ303020, JQ303022JQ303026 and JQ303028-JQ303034 (for Parma).

The results of our screening showed an overall rate of $3 \cdot 70 \%(49 / 1321)$ of HAstV infections, with a higher $(5 \cdot 8-7 \cdot 4 \%)$ range in Bari. In particular, during the 2-year study period, HAstVs were detected in Parma in $2.7 \%$ of the samples, in Bari in $6.9 \%$ and in Palermo in $1.9 \%$ of patients. A peak in HAstV prevalence was observed in Bari in 2008. Yearly prevalence, genotypes and lineages of the Italian HAstVs in the three study areas are reported in Table 1. The prevalence rates were similar to those reported in previous European studies [1, 10]. Agestratified serological investigations have revealed seroprevalence rates ranging form $10 \%$ to $91 \%$ in children, with exposure being highest to HAstV-1 and increasing with age [2]. Accordingly, it is likely that the majority of HAstV infections do not require hospitalization or medical care and may remain undetected. A phylogenetic tree based on the 348-bp fragment of the ORF2 was constructed to investigate the genetical relationships among the HAstVs detected in this study and to compare with other published sequences. Four different genotypes (HAstV-1, HAstV-2, HAstV-4, HAstV-5) were found to have been circulating in Italy during 2008-2009. HAstV-1 was predominant, accounting for $85.7 \%$ of the HAstV strains detected in the study, followed by HAstV-2 (8.16\%), HAstV-4 (6.1\%) and HAstV-5 $(2 \cdot 04 \%)$. Phylogenetical analysis allowed us to identify clearly the various lineages and genotypes (Fig. 1).

method were used to construct the phylogenetic tree. Bootstrap values above $70 \%$, estimated with 1000 pseudoreplicate datasets, are indicated at each node. Italian strains of this study are indicated as follows: $\boldsymbol{\square}, \mathrm{HAstV}$ from Bari; $\boldsymbol{\Delta}$, HAstV from Palermo; $\bullet$, HAstV from Parma. 
In $2008,58 \cdot 3 \%$ of HAstVs belonged to the HAstV-1d lineage, $27 \cdot 8 \%$ to HAstV-1a, $8 \cdot 3 \%$ to HAstV-4a and $2.7 \%$ to HAstV-2c and HAstV-5, while in 2009 HAstV-1a was predominant ( $80 \%)$, and apparently replaced the HAstV-1d lineage. Previous surveillance studies conducted in Palermo during 10 years have demonstrated the emergence and/or re-emergence of strains belonging to different HAstV lineages over time. In particular, HAstV-1d was first detected in Palermo in 1999, and was replaced by HAstV-1b in 2000. This lineage re-emerged in 2003-2005 and continued to be detected in 2008 along with HAstV-1a, which became predominant in 2009. Data are not available on the HAstV types circulating in other Italian areas in the same period and therefore this pattern could reflect either local or sampling variations. Furthermore, as the strains analysed in this study were collected from children with severe disease requiring hospitalization, they do not reflect the actual distribution of HAstV strains in the child population. However, our data are in agreement with other studies demonstrating that HAstV-1 is the prevalent type worldwide [2].

During this survey, an additional three other genotypes (HAstV-2, -4, -5) were also detected but at low frequency. HAstV-4 and HAstV-5 have been sporadically detected in different geographical areas, and are regarded as strains of minor epidemiological relevance $[1,4]$. On the other hand, HAstV-2 represented the second most prevalent type detected in this study and in 2009 it appeared to be more common than HAstV-1 in the area or Parma. These findings mirror the results of previous studies performed in Chile, Spain, Colombia and Brazil, describing the predominance of non-type $1 \mathrm{HAstV}$ in temporally and geographically restricted studies [11]. Upon sequence analysis, two different HAstV-2 lineages were identified. The type $2 \mathrm{c}$ lineage was first identified in Palermo in 2002 [7] and found to be circulating in 2008 in the local population, although at low frequency. The HAstV-2c strains circulating in 2008 showed $100 \%$ nucleotide identity to the old type $2 \mathrm{c}$ strains (PA65/02-like) (Fig. 1). However, only a small (348 bp) conserved portion at the 5'-end of ORF2 was analysed and used to predict HAstV types and lineages. Extending the analysis to the full-length ORF2 gene, including the hypervariable region of the capsid protein, could help assessing more properly the extent of genetic variation in these strains. Three type 2 strains detected in Parma in 2009 clustered together in a separate branch. Following previously defined criteria for lineage designation/distinction $[1,5]$ we propose that these strains are designated as a new lineage, namely HAstV-2d. These novel HAstV-2d strains shared $96 \cdot 7-98 \cdot 8 \%$ nucleotide identity to each other and displayed less than $93 \%$ nucleotide identity to HAstV-2a, $-2 \mathrm{~b}$ and $-2 \mathrm{c}$ strains. By observing in detail the nucleotide variation and fitting these changes into a polymorphism model for HAstV-2 lineages [8], a single nucleotide position $\left(\mathrm{B}_{381} \rightarrow \mathrm{A}\right)$ was able to differentiate the new HAstV-2d lineage from the other HAstV-2 lineages. An additional five silent nucleotide substitutions $\left(\mathrm{C}_{279} \rightarrow \mathrm{T}, \mathrm{C}_{471} \rightarrow \mathrm{T}, \mathrm{R}_{477} \rightarrow \mathrm{C}\right.$, $\mathrm{Y}_{480} \rightarrow \mathrm{A}, \mathrm{A}_{549} \rightarrow \mathrm{T}$ ), not included in the classification scheme, were helpful in distinguishing HAstV-2d from the other type 2 lineages in the short capsid region.

In conclusion, we established a nationwide surveillance system for HAstV in Italy including three different laboratories and adopted a common standardized diagnostic protocol demonstrating the cocirculation of several HAstV types and/or lineages. HAstV-1 was found to be predominant throughout the study period. A novel lineage, proposed as HAstV-2d, was found to have emerged in Parma in 2009. Investigating systematically the genetic variability of HAstVs will be important for understanding if periodic genotype shifts and genetic/antigenic drifts occur and if these changes may fit a model of evolution analogous to those observed for other antigenically/genetically heterogeneous viruses.

\section{ACKNOWLEDGEMENTS}

This investigation was supported by the grant 'Ricerca Scientifica FIL 2009', from the University of Parma, Italy.

\section{DECLARATION OF INTEREST}

None.

\section{REFERENCES}

1. Guix S, et al. Molecular epidemiology of astrovirus infection in Barcelona, Spain. Journal of Clinical Microbiology 2002; 40: 133-139.

2. Koopmans MP, et al. Age-stratified seroprevalence of neutralizing antibodies to astrovirus types 1 to 7 in humans in The Netherlands. Clinical and Diagnostic Laboratory Immunology 1998; 5: 33-37. 
3. Bányai K, et al. Detection of newly described astrovirus MLB1 in stool samples from children. Emerging Infectious Diseases 2010: 16; 169-170.

4. Mustafa H, Palombo EA, Bishop R. Epidemiology of astrovirus infection in young children hospitalized with acute gastroenteritis in Melbourn, Australia, over a period of four consecutive years, 1995 to 1998. Journal of Clinical Microbiology 2000; 38: 1058-1062.

5. Noel JS, et al. Typing of human astroviruses from clinical isolates by enzyme immunoassay and nucleotide sequencing. Journal of Clinical Microbiology 1995; 33: 797-801.

6. Gabbay YB, et al. Molecular epidemiology of astrovirus type 1 in Belem, Brazil, as an agent of infantile gastroenteritis, over a period of 18 years (1982-2000): identification of two possible new lineages. Virus Research 2007; 129: 166-174.

7. Colomba C, et al. Viral gastroenteritis in children hospitalised in Sicily, Italy. European Journal of
Clinical Microbiology and Infectious Diseases 2006: 25; 570-575.

8. De Grazia S, et al. Surveillance of human astrovirus circulation in Italy 2002-2005: emergence of linear 2c strains. Clinical Microbiology and Infectious Diseases 2011; 17: 97-101.

9. De Grazia S, et al. Molecular epidemiology of astrovirus infection in Italian children with gastroenteritis. Clinical Microbiology and Infectious Diseases 2004; 10: 1025-1029.

10. Jakab F, et al. One-year survey of astrovirus infection in children with gastroenteritis in a large hospital in Hungary: occurrence and genetic analysis of astroviruses. Journal of Medical Virology 2004; 74: 71-77.

11. Victoria M, et al. Genotypes and molecular epidemiology of human astroviruses in hospitalized children with acute gastroenteritis in Rio de Janeiro, Brazil. Journal of Medical Virology 2007; 79: 939-944. 